\author{
N. Kausar ${ }^{1, *}$, M. Munir ${ }^{2}$, M. Gulzar ${ }^{3}$, G. M. Addis ${ }^{4}$ \\ ${ }^{1}$ Department of Math and Stat, University of Agriculture, Faisalabad, Pakistan \\ ${ }^{2}$ Department of Mathematics, Government Postgraduate College, Abbottabad, Pakistan \\ ${ }^{3}$ Department of Mathematics, Government College University Faisalabad, Pakistan \\ ${ }^{4}$ Department of Mathematics, University of Gondar, Gondar, Ethiopia \\ (E-mail:kausar.nasreen57@gmail.com,dr.mohammadmunir@gpgc1-atd.edu.pk,98kohly@gmail.com, \\ gezahagne412@gmail.com)
}

\title{
Characterizing the Ordered AG-Groupoids Through the Properties of Their Different Classes of Ideals
}

\begin{abstract}
In this article, we have presented some important charcterizations of the ordered non-associative semigroups in relation to their ideals. We have initially characterized the ordered AG-groupoid through the properties of the their ideals, then we characterized the two important classes of these AG-groupoids, namely the regular and intragregular non-associative AG-groupoids. Our aim is also to encourage the research and the maturity of the associative algebraic structures by studying a class of non-associative and non-commutative algebraic structures called the ordered AG-groupoid.
\end{abstract}

Keywords: Ordered AG-groupoids, left (right, interior, quasi-, bi-, generalized bi-) ideals, regular (intraregular) ordered AG-groupoids.

\section{Introduction}

In 1972, a generalization of commutative semigroups has been established by Kazim et. al [1]. In ternary commutative law: $a b c=c b a$, they introduced the braces on the left side of this law and explored a new pseudo associative law, that is $(a b) c=(c b) a$. They have called the left invertive law of this law. A groupoid $S$ is said to be a left almost semigroup (abbreviated as LA-semigroup) if it satisfies the left invertive law : $(a b) c=(c b) a$. This structure is also known as Abel-Grassmann's groupoid (abbreviated as AG-groupoid) in [2]. An AG-groupoid is a midway structure between an abelian semigroup and a groupoid. Mushtaq et. al [3], investigated the concept of ideals in AG-groupoids.

In [4] (resp. [5]), a groupoid $S$ is said to be medial (resp. paramedial) if $(a b)(c d)=(a c)(b d)$ (resp. $(a b)(c d)=(d b)(c a))$. In [1], an AG-groupoid is medial, but in general an AG-groupoid needs not to be paramedial. Every AG-groupoid with left identity is paramedial by Protic et. al [2] and also satisfies $a(b c)=b(a c),(a b)(c d)=(d c)(b a)$.

In [6,7], if $(S, \cdot, \leq)$ is an ordered semigroup and $\emptyset \neq A \subseteq S$, we define a subset of $S$ as follows : $(A]=\{s \in S: s \leq a$ for some $a \in A\}$. A non-empty subset $A$ of $S$ is called a subsemigroup of $S$ if $A^{2} \subseteq A$.

A non-empty subset $A$ of $S$ is called a left (resp. right) ideal of $S$ if following hold (1) $S A \subseteq A$ (resp. $A S \subseteq A$ ). (2) If $a \in A$ and $b \in S$ such that $b \leq a$ implies $b \in A$. Equivalent definition: $A$ is called a left(resp. right) ideal of $S$ if $(A] \subseteq A$ and $S A \subseteq A$ (resp. $A S \subseteq A$ ).

A non-empty subset $A$ of $S$ is called an interior (resp. quasi-) ideal of $S$ if (1) $S A S \subseteq A$ (resp. $(A S] \cap(S A] \subseteq A)$. (2) If $a \in A$ and $b \in S$ such that $b \leq a$ implies $b \in A$.

A subsemigroup (A non-empty subset) $A$ of $S$ is called a bi- (generalized bi-) ideal of $S$ if (1) $A S A \subseteq A$. (2) If $a \in A$ and $b \in S$ such that $b \leq a$ implies $b \in A$. Every bi-ideal of $S$ is a generalized bi-ideal of $S$.

\footnotetext{
${ }^{*}$ Corresponding author.

E-mail: kausar.nasreen57@gmail.com
} 
In $[7,8]$, an ordered semigroup is said to be regular if for every $a \in S$, there exists an element $x \in S$ such that $a \leq a x a$. Equivalent definitions are as follows: $(1) A \subseteq(A S A]$ for every $A \subseteq S$. (2) $a \in(a S a]$ for every $a \in S$.

In $[9,10]$, an ordered semigroup $S$ is intra-regular if for every $a \in S$ there exist elements $x, y \in S$ such that $a \leq x a^{2} y$. Equivalent definitions are as follows: (1) $A \subseteq\left(S A^{2} S\right]$ for every $A \subseteq S$. (2) $a \in\left(S a^{2} S\right]$ for every $a \in S$.

We will define left (right, interior, quasi-, bi-, generalized bi-) ideals in ordered AG-groupoids. We will establish a study by discussing the different properties of such ideals. We will also characterize regular (resp. intra-regular, both regular and intra-regular) ordered AG-groupoids by the properties of left (right, quasi-, bi-, generalized bi-) ideals.

\section{Ideals in Ordered AG-groupoids}

An ordered AG-groupoid $S$, is a partially ordered set, at the same time an AG-groupoid such that $a \leq b$, implies $a c \leq b c$ and $c a \leq c b$ for all $a, b, c \in S$. Two conditions are equivalent to the one condition $(c a) d \leq(c b) d$ for all $a, b, c, d \in S$.

Example 1. Consider a set $S=\{e, f, a, b, c\}$ with the following multiplication "." and order relation " $\leq "$

\begin{tabular}{l|lllll}
$\cdot$ & $e$ & $f$ & $a$ & $b$ & $c$ \\
\hline$e$ & $e$ & $f$ & $a$ & $b$ & $c$ \\
$f$ & $f$ & $f$ & $f$ & $b$ & $c$ \\
$a$ & $a$ & $f$ & $c$ & $b$ & $c$ \\
$b$ & $c$ & $c$ & $c$ & $f$ & $b$ \\
$c$ & $b$ & $b$ & $b$ & $c$ & $f$
\end{tabular}

$\leq=\{(e, e),(e, a),(e, b),(e, c),(f, f),(f, b),(f, c),(a, a),(a, c),(b, b),(b, c),(c, c)\}$.

Then $(S, \cdot, \leq)$ is an ordered AG-groupoid with left identity $e$.

For $\emptyset \neq A \subseteq S$, we define a subset $(A]=\{s \in S: s \leq a$ for some $a \in A\}$ of $S$ and obviously $A \subseteq(A]$. For $\emptyset \neq A, B \subseteq S$, then $((A]]=(A],(A](B] \subseteq(A B],((A](B]]=(A B]$, if $A \subseteq B$, then $(A] \subseteq(B],(A \cap B] \neq(A] \cap(B]$, in general.

For $\emptyset \neq A \subseteq S$. Then $A$ is called an ordered AG-subgroupoid of $S$ if $A^{2} \subseteq A$. $A$ is called a left (resp. right) ideal of $S$ if the following hold (1) $S A \subseteq A$ (resp. $A S \subseteq A$ ). (2) If $a \in A$ and $b \in S$ such that $b \leq a$ implies $b \in A$. $A$ is called an ideal of $S$ if $A$ is both a left and a right ideal of $S$.

We denote by $L(a), R(a), I(a)$ the left ideal, the right ideal and the ideal of $S$, respectively, generated by $a$. we have $L(a)=\{s \in S: s \leq a$ or $s \leq x a$ for some $x \in S\}=(a \cup S a], R(a)=(a \cup a S]$, $I(a)=(a \cup S a \cup a S \cup(S a) S]$.

A non-empty subset $A$ of an ordered AG-groupoid $S$ is called an interior (resp. quasi-) ideal of $S$ if (1) $(S A) S \subseteq A$ (resp. $(A S] \cap(S A] \subseteq A$ ). (2) If $a \in A$ and $b \in S$ such that $b \leq a$ implies $b \in A$.

An AG-subgroupoid $A$ of $S$ is called a bi-ideal of $S$ if (1) $(A S) A \subseteq A$. (2) If $a \in A$ and $b \in S$ such that $b \leq a$ implies $b \in A$. A non-empty subset $A$ of $S$ is called generalized bi-ideal of $S$ if (1) $(A S) A \subseteq A$. (2) If $a \in A$ and $b \in S$ such that $b \leq a$ implies $b \in A$.

Now we give the imperative properties of such ideals of an ordered AG-groupoid $S$, which will be play a vital rule in the later sections. Specifically we show:

(1) Let $S$ be an ordered AG-groupoid with left identity $e$. Then every right ideal of $S$ is a ideal of $S$.

(2) Let $S$ be an ordered AG-groupoid with left identity $e$, such that $(x e) S=x S$ for all $x \in S$. Then every quasi-ideal of $S$ is a bi-ideal of $S$.

Lemma 1. Let $S$ be an ordered AG-groupoid with left identity $e$. Then $S S=S$ and $e S=S=S e$.

Proof: Since $S S \subseteq S$ and $x=e x \in S S$, i.e., $S \subseteq S S$, thus $S S=S$. Obviously, $e S=S$ and $S e=(S S) e=(e S) S=S S=S$. 
Lemma 2. Let $S$ be an odered AG-groupoid with left identity $e$ and $a \in S$. Then $S a$ is a smallest left ideal of $S$ containing $a$.

Proof: Let $x \in S a$ and $s \in S$, this implies that $x=s_{1} a$, where $s_{1} \in S$. Now

$$
\begin{aligned}
s x & =s\left(s_{1} a\right)=(e s)\left(s_{1} a\right)=\left(\left(s_{1} a\right) s\right) e=\left(\left(s_{1} a\right)(e s)\right) e \\
& =\left(\left(s_{1} e\right)(a s)\right) e=(e(a s))\left(s_{1} e\right)=(a s)\left(s_{1} e\right)=\left(\left(s_{1} e\right) s\right) a \in S a .
\end{aligned}
$$

Thus $s x \in S a$ and $(S a] \subseteq S a$. Since $a=e a \in S a$, hence $S a$ is a left ideal of $S$ containing $a$. Let $I$ be another left ideal of $S$ containing $a$. Since $s a \in I$, because $I$ is a left ideal of $S$. But $s a \in S a$, this means that $S a \subseteq I$. Therefore $S a$ is a smallest left ideal of $S$ containing $a$.

Lemma 3. Let $S$ be an odered AG-groupoid with left identity $e$ and $a \in S$. Then $a S$ is a left ideal of $S$.

Proof: Straight forward.

Proposition 1. Let $S$ be an ordered AG-groupoid with left identity $e$ and $a \in S$. Then $a S \cup S a$ is a smallest right ideal of $S$ containing $a$.

Proof: Let $x \in a S \cup S a$. We have to show that $(a S \cup S a) S \subseteq a S \cup S a$. Now

$$
\begin{aligned}
(a S \cup S a) S & =(a S) S \cup(S a) S=(S S) a \cup(S a)(e S) \\
& \subseteq S a \cup(S e)(a S)=S a \cup S(a S) \\
& =S a \cup a(S S) \subseteq S a \cup a S=a S \cup S a .
\end{aligned}
$$

Thus $(a S \cup S a) S \subseteq a S \cup S a$ and $(a S \cup S a] \subseteq a S \cup S a$. Therefore $a S \cup S a$ is a right ideal of $S$. Since $a \in S a$, i.e., $a \in a S \cup S a$. Let $I$ be another right ideal of $S$ containing $a$. Now $a S \in I S \subseteq I$ and $S a=(S S) a=(a S) S \in(I S) S \subseteq I S \subseteq I$, i.e., $a S \cup S a \subseteq I$. Hence $a S \cup S a$ is a smallest right ideal of $S$ containing $a$.

Lemma 4. Let $S$ be an ordered AG-groupoid with left identity $e$. Then every right ideal of $S$ is an ideal of $S$.

Proof: Let $R$ be a right ideal of $S$ and $r \in R, s \in S$. Now $s r=(e s) r=(r s) e \in(R S) S \subseteq R S \subseteq R$. Thus $S R \subseteq R$ and $(R] \subseteq R$. Hence $R$ is an ideal of $S$.

Lemma 5. Let $S$ be an ordered AG-groupoid with left identity $e$ such that $(x e) S=x S$ for all $x \in S$. Then $(A S) S \subseteq A S$ and $(A S] S \subseteq(A S]$.

Proof: Since

$$
\begin{aligned}
(A S) S & =(A S)(e S)=(A e)(S S) \subseteq(A e) S=A S . \\
\text { and }(A S] S & =(A S](S] \subseteq((A S) S] \subseteq(A S] .
\end{aligned}
$$

Remark 1. Let $S$ be an ordered AG-groupoid with left identity $e$ such that $(x e) S=x S$ for all $x \in S$, then $(A S]$ is an ideal of $S$.

Let $S$ be an ordered AG-groupoid with left identity $e$ such that $(x e) S=x S$ for all $x \in S$ and $A, B \subseteq S$. Then $(A S)(B S) \subseteq(A B) S$ and $(A S](B S] \subseteq((A B) S]$. Similarly $(S A)(S B) \subseteq S(A B)$ and $(S A](S B] \subseteq(S(A B)]$.

In general for $A_{1}, A_{2}, \ldots, A_{n} \subseteq S$, then $\left(A_{1} S\right)\left(A_{2} S\right) \ldots\left(A_{n} S\right) \subseteq\left(A_{1} A_{2}, \ldots, A_{n}\right) S$ and $\left(A_{1} S\right]\left(A_{2} S\right] \ldots$ $\left(A_{n} S\right] \subseteq\left(\left(A_{1} A_{2}, \ldots, A_{n}\right) S\right]$.

Similarly, $\left(S A_{1}\right)\left(S A_{2}\right) \ldots\left(S A_{n}\right) \subseteq S\left(A_{1} A_{2}, \ldots, A_{n}\right)$ and $\left(S A_{1}\right]\left(S A_{2}\right] \ldots .\left(S A_{n}\right] \subseteq\left(S\left(A_{1} A_{2}, \ldots, A_{n}\right)\right]$.

Lemma 6 . Let $S$ be an ordered AG-groupoid. $A$ is a right ideal of $S$ and $B$ is a right ideal of $A$, then $(B]=B$.

Proof: Since $(B]=\{s \in S \mid s \leq b$ for some $b \in B\}$ and $s \in(B]$, this implies that there exists an element $s \in S$ such that $s \leq b$ for some $b \in B \subseteq A$. Thus $S \ni s \leq b \in A$. Now $A \ni s \leq b \in B$ and $B$ is a right ideal of $A$, i.e., $s \in B$, so $(B] \subseteq B$. Since $B \subseteq(B]$, thus $(B]=B$. 
Proposition 2. Let $S$ be an ordered AG-groupoid with left identity $e$ such that $(x e) S=x S$ for all $x \in S$. $A$ is a right ideal of $S$ and $B$ is a right ideal of $A$ such that $\left(B^{2}\right]=B$. Then $B$ is an ideal of $S$.

Proof: We have to show that $B$ is a right ideal of $S$. Now

$$
\begin{aligned}
B S & =\left(B^{2}\right] S=\left(B^{2}\right](S] \subseteq\left(B^{2} S\right]=((B B) S] \\
& =((S B) B] \subseteq((S B) A]=((S B)(e A)] \\
& =((S e)(B A)]=(B((S e) A)]=(B((A e) S)] \\
& =(B(A S)] \subseteq(B A] \subseteq(B]=B \text { by the Lemma } 6 .
\end{aligned}
$$

Thus $B S \subseteq B$ and $(B] \subseteq B$, i.e., $B$ is a right ideal of $S$. Hence $B$ is an ideal of $S$ by the Lemma 4 .

Lemma \%. Let $S$ be an ordered AG-groupoid. $A$ is a left ideal of $S$ and $B$ is a left ideal of $A$, then $(B]=B$.

Proof: Same as Lemma 6.

Proposition 3. Let $S$ be an ordered AG-groupoid with left identity $e$. $A$ is a left ideal of $S$ and $B$ is a left ideal of $A$ such that $\left(B^{2}\right]=B$. Then $B$ is left ideal of $S$.

Proof: We have to show that $B$ is a left ideal of $S$. Now

$$
\begin{aligned}
S B & =S\left(B^{2}\right]=(S]\left(B^{2}\right] \subseteq\left(S B^{2}\right]=(S(B B)] \\
& =((S e)(B B)]=((S B)(e B)] \\
& \subseteq((S A)(e B)] \subseteq(A B] \subseteq(B]=B, \quad \text { by the Lemma } 7 .
\end{aligned}
$$

Thus $S B \subseteq B$ and $(B] \subseteq B$. Hence $B$ is a left ideal of $S$.

Lemma 8. Every two-sided ideal of $S$ is an interior ideal of $S$.

Proof: Straight forward.

Proposition 4. Let $S$ be an ordered AG-groupoid with left identity $e$. Then any non-empty subset $I$ of $S$ is an ideal of $S$ if and only if $I$ is an interior ideal of $S$.

Proof: Suppose that $I$ is an interior ideal of $S$. Let $i \in I$ and $s \in S$. Now $i s=(e i) s \in(S I) S \subseteq I$, this implies that $I S \subseteq I$ and $(I] \subseteq I$, i.e., $I$ is a right ideal of $S$. Hence $I$ is an ideal of $S$ by the Lemma 4. Converse is true by the Lemma 8.

Lemma 9. Every right (two-sided) ideal of $S$ is a bi-ideal of $S$.

Proof: Straight forward.

Lemma 10. Every bi-ideal of $S$ is a generalized bi-ideal of $S$.

Proof: Obvious.

Lemma 11. Every left (right, two-sided) ideal of $S$ is a quasi-ideal of $S$.

Proof: Let $I$ be a right ideal of $S$. Now $(I S] \cap(S I] \subseteq(I S] \subseteq(I] \subseteq I$ and $(I] \subseteq I$. Thus $I$ is a quasi-ideal of $S$.

Proposition 5. Every quasi-ideal of $S$ is an ordered AG-subgroupoid of $S$.

Proof: Suppose that $I$ is a quasi-ideal of $S$. Now $I I \subseteq I S \subseteq(I](S] \subseteq(I S]$ and $I I \subseteq S I \subseteq(S](I] \subseteq$ $\subseteq(S I]$, i.e., $I^{2}=I I \subseteq(I S] \cap(S I] \subseteq I$. Hence $I$ is an AG-subgroupoid of $S$.

Proposition 6 . Let $R$ be a right ideal and $L$ be a left ideal of an ordered AG-groupoid $S$, respectively. Then $R \cap L$ is a quasi-ideal of $S$.

Proof: Since $((R \cap L) S] \cap(S(R \cap L)] \subseteq(R S] \cap(S L] \subseteq(R] \cap(L] \subseteq R \cap L$ and $(R \cap L]=R \cap L$. Thus $R \cap L$ is a quasi-ideal of $S$.

Lemma 12. Let $S$ be an ordered AG-groupoid with left identity $e$ such that $(x e) S=x S$ for all $x \in S$. Then every quasi-ideal of $S$ is a bi-ideal of $S$.

Proof: Let $Q$ be a quasi-ideal of $S$. Now $(Q S) Q \subseteq(S S) Q \subseteq S Q \subseteq(S Q]$ and $(Q S) Q \subseteq(Q S) S=$ $=(Q S)(e S)=(Q e)(S S)=(Q e) S=Q S \subseteq(Q S]$, thus $(Q S) Q \subseteq(Q S] \cap(S Q] \subseteq Q$. Therefore $(Q S) Q \subseteq Q$ and $(Q] \subseteq Q$. Hence $Q$ is a bi-ideal of $S$. 


\section{Regular Ordered AG-groupoids}

An ordered AG-groupoid $S$ is called regular if for every $a \in S$, there exists an element $x \in S$ such that $a \leq(a x) a$. Equivalent definitions are as follows:

(1) $A \subseteq((A S) A]$ for every $A \subseteq S$.

(2) $a \in((a S) a]$ for every $a \in S$.

An ideal $I$ of an ordered AG-groupoid $S$ is called idempotent if $\left(I^{2}\right]=I$.

In this section, we characterize regular ordered AG-groupoids by the properties of (left, right, quasi-, bi-, generalized bi-) ideals.

Lemma 13. Every right ideal of a regular ordered AG-groupoid $S$

Proof: Let $R$ be a right ideal of $S$. Let $r \in R$ and $a \in S$, this implies that there exists an element $x \in S$ such that $a \leq(a x) a$. Now $a r \leq((a x) a) r=(r a)(a x) \in R S \subseteq R$, thus $S R \subseteq R$ and $(R]=R$. Hence $R$ is an ideal of $S$.

Lemma 14. Every ideal of a regular ordered AG-groupoid $S$ is an idempotent.

Proof: Suppose that $I$ is an ideal of $S$ and $\left(I^{2}\right]=(I I] \subseteq(I]=I$. Let $a \in I$, this mean that there exists an element $x \in S$ such that $a \leq(a x) a$. Now $a \leq(a x) a \in(I S) I \subseteq I I=I^{2}$, i.e., $I \subseteq\left(I^{2}\right]$. Therefore $\left(I^{2}\right]=I$.

Remark 2. Every right ideal of a regular ordered AG-groupoid $S$ is an idempotent.

Proposition 7. Let $S$ be a regular ordered AG-groupoid. Then any non-empty subset $I$ of $S$ is an ideal of $S$ if and only if $I$ is an interior ideal of $S$.

Proof: Assume that $I$ is an interior ideal of $S$. Let $a \in I$ and $s \in S$, then there exists an element $x \in S$, such that $a \leq(a x) a$. Now as $\leq((a x) a) s=(s a)(a x) \in(S I) S \subseteq I$. Thus $I S \subseteq I$ and $(I]=I$, i.e., $I$ is a right ideal of $S$. Hence $I$ is an ideal of $S$ by the Lemma 4 . Converse is true by the Lemma 13.

Proposition 8. Let $S$ be a regular ordered AG-groupoid with left identity e. Then $(I S] \cap(S I]=I$, for every right ideal $I$ of $S$.

Proof: Let $I$ be an ideal of $S$. This implies that $(I S] \cap(S I] \subseteq I$, because every ideal of $S$ is a quasi-ideal of $S$. Let $a \in I$, this means that there exists an element $x \in S$ such that $a \leq(a x) a$. Now $a \leq(a x) a \in(I S) I \subseteq I I \subseteq I S$, i.e., $I \subseteq(I S]$. Now $a \leq(a x) a=(a x)(e a)=(a e)(x a) \in I I \subseteq S I$, i.e., $I \subseteq(S I]$. Thus $I \subseteq(I S] \cap(S I]$. Hence $(I S] \cap(S I]=I$.

Lemma 15. Let $S$ be a regular ordered AG-groupoid. Then $(R L]=R \cap L$, for every right ideal $R$ and every left ideal $L$ of $S$.

Proof: Since $(R L] \subseteq(R S] \subseteq(R]=R$ and $(R L] \subseteq(S L] \subseteq(L]=L$, i.e., $(R L] \subseteq R \cap L$. Let $a \in R \cap L$, this implies that there exists an element $x \in S$ such that $a \leq(a x) a$. Now $a \leq(a x) a \in(R S) L \subseteq R L$, i.e., $R \cap L \subseteq(R L]$. Therefore $(R L]=R \cap L$.

Theorem 1. Let $S$ be an ordered AG-groupoid with left identity $e$ such that $(x e) S=x S$ for all $x \in S$. Then the following conditions are equivalent.

(1) $S$ is a regular.

(2) $R \cap L=(R L]$ for every right ideal $R$ and every left ideal $L$ of $S$.

(3) $Q=((Q S) Q]$ for every quasi-ideal $Q$ of $S$.

Proof: Suppose that (1) holds. Let $Q$ be a quasi-ideal of $S$ and $a \in Q$, this implies that there exists an element $x \in S$ such that $a \leq(a x) a$. Now $a \leq(a x) a \in(Q S) Q$, i.e., $Q \subseteq((Q S) Q] \subseteq(Q]=Q$, because every quasi-ideal of $S$ is a bi-ideal of $S$. Hence $Q=((Q S) Q]$, i.e., $(1) \Rightarrow(3)$. Assume that (3) holds, let $R$ be a right ideal and $L$ be a left ideal of $S$. Then $R$ and $L$ be quasi-ideals of $S$ by the Lemma 11 , so $R \cap L$ be a quasi-ideal of $S$. Now $R \cap L=(((R \cap L) S)(R \cap L)] \subseteq((R S) L] \subseteq(R L]$. Since $(R L] \subseteq R \cap L$, so $(R L]=R \cap L$, i.e., (3) $\Rightarrow(2)$. Suppose that (2) is true, let $a \in S$, then $S a$ is a left ideal of $S$ containing $a$ by the Lemma 2 and $a S \cup S a$ is a right ideal of $S$ containing $a$ by the 
Proposition 1. By (2),

$$
\begin{aligned}
(a S \cup S a) \cap S a & =((a S \cup S a)(S a)]=((a S)(S a) \cup(S a)(S a)] . \\
(S a)(S a) & =((S e) a)(S a)=((a e) S)(S a)=(a S)(S a) .
\end{aligned}
$$

Thus

$$
\begin{aligned}
(a S \cup S a) \cap S a & =((a S)(S a) \cup(S a)(S a)] \\
& =((a S)(S a) \cup(a S)(S a)]=((a S)(S a)] .
\end{aligned}
$$

Since $a \in(a S \cup S a) \cap S a$, Implies $a \in((a S)(S a)]$. Then $a \leq(a x)(y a)=((y a) x) a=(((e y) a) x) a=$ $=(((a y) e) x) a=((x e)(a y)) a=(a((x e) y)) a \in(a S) a$ for any $x, y \in S$, i.e., $a \in((a S) a]$. Hence $a$ is regular, so $S$ is a regular, i.e., $(2) \Rightarrow(1)$.

Theorem 2. Let $S$ be an ordered AG-groupoid with left identity $e$ such that $(x e) S=x S$ for all $x \in S$. Then the following conditions are equivalent.

(1) $S$ is a regular.

(2) $Q=((Q S) Q]$ for every quasi-ideal $Q$ of $S$.

(3) $B=((B S) B]$ for every bi-ideal $B$ of $S$.

(4) $G=((G S) G]$ for every generalized bi-ideal $G$ of $S$.

Proof: $(1) \Rightarrow(4)$, is obvious. (4) $\Rightarrow(3)$, since every bi-ideal of $S$ is a generalized bi-ideal of $S$ by the Lemma 10. (3) $\Rightarrow(2)$, since every quasi-ideal of $S$ is bi-ideal of $S$ by the Lemma 12 . (2) $\Rightarrow(1)$, by the Theorem 1 .

Theorem 3. Let $S$ be an ordered AG-groupoid with left identity e such that $(x e) S=x S$ for all $x \in S$. Then the following conditions are equivalent.

(1) $S$ is a regular.

(2) $Q \cap I=((Q I) Q]$ for every quasi-ideal $Q$ and every ideal $I$ of $S$.

(3) $B \cap I=((B I) B]$ for every bi-ideal $B$ and every ideal $I$ of $S$.

(4) $G \cap I=((G I) G]$ for every generalized bi-ideal $G$ and every ideal $I$ of $S$.

Proof: Suppose that (1) is true. Let $G$ be a generalized bi-ideal and $I$ be an ideal of $S$. Now $((G I) G] \subseteq((S I) S] \subseteq(I]=I$ and $((G I) G] \subseteq((G S) G] \subseteq(G]=G$, thus $((G I) G] \subseteq G \cap I$. Let $a \in G \cap I$, this means that there exists an element $x \in S$ such that $a \leq(a x) a$. Now $a \leq(a x) a=$ $=(((a x) a) x) a=((x a)(a x)) a=(a((x a) x)) a \in(G I) G$, thus $G \cap I \subseteq((G I) G]$. Hence $G \cap I=((G I) G]$, i.e., $(1) \Rightarrow(4)$. (4) $\Rightarrow(3)$, since every bi-ideal of $S$ is a generalized bi-ideal of $S$ by the Lemma 10 . $(3) \Rightarrow(2)$, since every quasi-ideal of $S$ is a bi-ideal of $S$ by the Lemma 12. Assume that (2) is true. Now $Q \cap S=((Q S) Q]$, i.e., $Q=((Q S) Q]$, where $Q$ is a quasi-ideal of $S$. Hence $S$ is a regular by the Theorem 1.

Theorem 4. Let $S$ be an ordered AG-groupoid with left identity $e$ such that $(x e) S=x S$ for all $x \in S$. Then the following conditions are equivalent.

(1) $S$ is a regular.

(2) $R \cap Q \subseteq(R Q]$ for every quasi-ideal $Q$ and every right ideal $R$ of $S$.

(3) $R \cap B \subseteq(R B]$ for every bi-ideal $B$ and every right ideal $R$ of $S$.

(4) $R \cap G \subseteq(R G]$ for every generalized bi-ideal $G$ and every right ideal $R$ of $S$.

Proof: (1) $\Rightarrow(4)$, is obvious. (4) $\Rightarrow(3)$, since every bi-ideal of $S$ is a generalized bi-ideal of $S$. $(3) \Rightarrow(2)$, since every quasi-ideal of $S$ is a bi-ideal of $S$ by the Lemma 12. . Suppose that (2) is true. Now $R \cap Q=Q \cap R \subseteq(R Q]$, where $Q$ is a left ideal and $R$ is right ideal of $S$, because every left ideal of $S$ is a quasi-ideal of $S$. Since $(R Q] \subseteq R \cap Q$, thus $R \cap Q=(R Q]$. Hence $S$ is a regular, by the Theorem 1. 


\section{Intra-regular Ordered AG-groupoids}

An ordered AG-groupoid $S$ is called intra-regular if for every $a \in S$, there exist elements $x, y \in S$ such that $a \leq\left(x a^{2}\right) y$. Equivalent definitions are as follows:

(1) $A \subseteq\left(\left(S A^{2}\right) S\right]$ for every $A \subseteq S$.

(2) $a \in\left(\left(S a^{2}\right) S\right]$ for every $a \in S$.

In this section, we characterize intra-regular ordered AG-groupoids by the properties of (left, right, quasi-, bi-, generalized bi-) ideals.

Lemma 16. Every left (right) ideal of an intra-regular ordered AG-groupoid $S$ is an ideal of $S$.

Proof: Let $R$ be a right ideal of $S$. Let $r \in R$ and $a \in S$, this implies that there exist elements $x, y \in S$ such that $a \leq\left(x a^{2}\right) y$. Now ar $\leq\left(\left(x a^{2}\right) y\right) r=(r y)\left(x a^{2}\right) \in R S \subseteq R$. Thus $S R \subseteq R$ and $(R] \subseteq R$. Hence $R$ is an ideal of $S$.

Lemma 17. Every ideal of an intra-regular ordered AG-groupoid $S$ with left identity $e$, is an idempotent.

Proof: Suppose that $I$ is an ideal of $S$ and $\left(I^{2}\right]=(I I] \subseteq(I]=I$. Let $a \in I$, this means that there exist elements $x, y \in S$ such that $a \leq\left(x a^{2}\right) y$. Now

$$
\begin{aligned}
a & \leq\left(x a^{2}\right) y=(x(a a)) y=(a(x a)) y \\
& =(a(x a))(e y)=(a e)((x a) y)=(x a)((a e) y) \in I I .
\end{aligned}
$$

Thus $a \in(I I]=\left(I^{2}\right]$. Therefore $\left(I^{2}\right]=I$.

Proposition 9. Let $S$ be an intra-regular ordered AG-groupoid with left identity $e$. Then any nonempty subset $I$ of $S$ is an ideal of $S$ if and only if $I$ is an interior ideal of $S$.

Proof: Assume that $I$ is an interior ideal of $S$. Let $i \in I$ and $a \in S$, then there exist elements $x, y \in S$ such that $x \leq\left(y x^{2}\right) z$. Now

$$
\begin{aligned}
i a & \leq i\left(\left(x a^{2}\right) y\right)=i((x(a a)) y) \\
& =i((a(x a)) y)=i((a(x a))(e y)) \\
& =i((a e)((x a) y))=i((x a)((a e) y)) \\
& =(x a)(i((a e) y))=(x i)(a((a e) y)) \in(S I) S \subseteq I .
\end{aligned}
$$

Thus $I S \subseteq I$ and $(I] \subseteq I$, i.e., $I$ is a right ideal of $S$. So $I$ is an ideal of $S$ by the Lemma 16 . Converse is obvious.

Lemma 18. Let $S$ be an intra-regular ordered AG-groupoid with left identity $e$. Then $L \cap R \subseteq(L R]$ for every left ideal $L$ and every right ideal $R$ of $S$.

Proof: Let $a \in L \cap R$, where $L$ is a left ideal and $R$ is a right ideal of $S$, respectively, this implies that there exist elements $x, y \in S$ such that $a \leq\left(x a^{2}\right) y$. Now

$$
\begin{aligned}
a & \leq\left(x a^{2}\right) y=(x(a a)) y=(a(x a)) y=(a(x a))(e y) \\
& =(a e)((x a) y)=(x a)((a e) y) \in L R . \\
& \Rightarrow L \cap R \subseteq(L R] .
\end{aligned}
$$

Theorem 5. Let $S$ be an ordered AG-groupoid with left identity $e$ such that $(x e) S=x S$ for all $x \in S$. Then the following conditions are equivalent.

(1) $S$ is an intra-regular.

(2) $L \cap R \subseteq(L R]$ for every left ideal $L$ and every right ideal $R$ of $S$.

Proof: Since $(1) \Rightarrow(2)$ holds by the Lemma 18. Suppose that (2) holds and $a \in S$, then $S a$ is a left ideal of $S$ containing $a$ and $a S \cup S a$ is a right ideal of $S$ containing $a$. By our supposition

$$
\begin{aligned}
S a \cap(a S \cup S a) & \subseteq((S a)(a S \cup S a)]=((S a)(a S) \cup(S a)(S a)] . \\
(S a)(a S) & =(S a)((e a) S)=(S a)((S a) e)=(S a)((S a)(e e)) \\
& =(S a)((S e)(a e))=(S a)(S(a e))=(S a)(S a) .
\end{aligned}
$$


Thus

$$
\begin{aligned}
(a S \cup S a) \cap S a & \subseteq((S a)(a S) \cup(S a)(S a)] \\
& =((S a)(S a) \cup(S a)(S a)] \\
& =((S a)(S a)]=\left(S^{2} a^{2}\right]=\left(S a^{2}\right] \\
& =\left(S\left(a^{2} e\right)\right]=\left((S S)\left(a^{2} e\right)\right]=\left((e S)\left(a^{2} S\right)\right]=\left(S\left(a^{2} S\right)\right] \\
& =\left(a^{2}(S S)\right]=\left(\left(e a^{2}\right)(S S)\right]=\left(\left(S a^{2}\right)(S e)\right]=\left(\left(S a^{2}\right) S\right] .
\end{aligned}
$$

Since $a \in(a S \cup S a) \cap S a$, implies $a \in\left(\left(S a^{2}\right) S\right]$, thus $a$ is an intra regula. Hence $S$ is an intra-regular, i.e., $(2) \Rightarrow(1)$.

Theorem 6. Let $S$ be an ordered AG-groupoid with left identity $e$ such that $(x e) S=x S$ for all $x \in S$. Then the following conditions are equivalent.

(1) $S$ is an intra-regular.

(2) $Q \cap I=((Q I) Q]$ for every quasi-ideal $Q$ and every ideal $I$ of $S$.

(3) $B \cap I=((B I) B]$ for every bi-ideal $B$ and every ideal $I$ of $S$.

(4) $G \cap I=((G I) G]$ for every generalized bi-ideal $G$ and every ideal $I$ of $S$.

Proof: Suppose that (1) holds. Let $a \in G \cap I$, where $G$ is a generalized bi-ideal and $I$ is an ideal of $S$, this implies that there exist elements $x, y \in S$ such that $a \leq\left(x a^{2}\right) y$. Now

$$
\begin{aligned}
a & \leq\left(x a^{2}\right) y=(x(a a)) y=(a(x a)) y=(y(x a)) a . \\
y(x a) & \leq y\left(x\left(\left(x a^{2}\right) y\right)\right)=y\left(\left(x a^{2}\right)(x y)\right)=\left(x a^{2}\right)(y(x y)) \\
& =\left(x a^{2}\right)\left(x y^{2}\right)=(x(a a)) m, \text { say } x y^{2}=m \\
& =(a(x a)) m=(m(x a)) a . \\
m(x a) & \leq m\left(x\left(\left(x a^{2}\right) y\right)\right)=m\left(\left(x a^{2}\right)(x y)\right)=\left(x a^{2}\right)(m(x y)) \\
& =(x(a a)) n, \text { say } m(x y)=n \\
& =(a(x a)) n=(n(x a)) a \\
& =v a, \text { say } n(x a)=v . \\
& \Rightarrow y(x a)=(m(x a)) a=(v a) a=(v a)(e a)=(v e)(a a)=a((v e) a) .
\end{aligned}
$$

Thus $a \leq\left(x a^{2}\right) y=(y(x a)) a=(a((v e) a)) a \in(G I) G$. This means that $a \in((G I) G]$, i.e., $G \cap I \subseteq((G I) G]$. Now $((G I) G] \subseteq((S I) S] \subseteq(I]=I$ and $((G I) G] \subseteq((G S) G] \subseteq(G]=G$, thus $((G I) G] \subseteq G \cap I$. Hence $G \cap I=((G I) G]$, i.e., $(1) \Rightarrow(4)$. (4) $\Rightarrow(3)$, every bi-ideal of $S$ is a generalized bi-ideal of $S$ by the Lemma 10. (3) $\Rightarrow(2)$, every quasi-ideal of $S$ is a bi-ideal of $S$ by the Lemma 12. Assume that (2) is true and let $R$ be a right ideal and $I$ be a two-sided ideal of $S$. Now $I \cap R=((R I) R] \subseteq((S I) R] \subseteq(I R]$, since every right ideal of $S$ is a quasi-ideal of $S$. Therefore $S$ is an intra-regular by the Theorem 5 , i.e., $(2) \Rightarrow(1)$.

Theorem 7. Let $S$ be an ordered AG-groupoid with left identity $e$ such that $(x e) S=x S$ for all $x \in S$. Then the following conditions are equivalent.

(1) $S$ is an intra-regular.

(2) $L \cap Q \subseteq(L Q]$ for every quasi-ideal $Q$ and every left ideal $L$ of $S$.

(3) $L \cap B \subseteq(L B]$ for every bi-ideal $B$ and every left ideal $L$ of $S$.

(4) $L \cap G \subseteq(L G]$ for every generalized bi-ideal $G$ and every left ideal $L$ of $S$.

Proof: Suppose that (1) holds. Let $a \in L \cap G$, where $L$ is a left ideal and $G$ is a generalized bi-ideal of $S$, this means that there exist elements $x, y \in S$ such that $a \leq\left(x a^{2}\right) y$. Now $a \leq\left(x a^{2}\right) y=(x(a a)) y=$ $=(a(x a)) y=(y(x a)) a \in L G$, i.e., $a \in(L G]$. Thus $L \cap G \subseteq(L G]$, i.e., (1) $\Rightarrow(4)$. (4) $\Rightarrow(3)$, every bi-ideal of $S$ is a generalized bi-ideal of $S$. (3) $\Rightarrow(2)$, every quasi-ideal of $S$ is a bi-ideal of $S$. Assume that (2) is true and let $R$ be a right ideal of $S$ and $L$ be a left ideal of $S$. Now $L \cap R \subseteq(L R]$, where $R$ is a quasi-ideal of $S$. Hence $S$ is an intra-regular by the Theorem 5, i.e., (2) $\Rightarrow(1)$. 
Theorem 8. Let $S$ be an ordered AG-groupoid with left identity $e$ such that $(x e) S=x S$ for all $x \in S$. Then the following conditions are equivalent.

(1) $S$ is an intra-regular.

(2) $L \cap Q \cap R \subseteq((L Q) R]$ for every quasi-ideal $Q$, every right ideal $R$ and every left ideal $L$ of $S$.

(3) $L \cap B \cap R \subseteq((L B) R]$ for every bi-ideal $B$, every right ideal $R$ and every left ideal $L$ of $S$.

(4) $L \cap G \cap R \subseteq((L G) R]$ for every generalized bi-ideal $G$, every right ideal $R$ and every left ideal $L$ of $S$.

Proof: Suppose that (1) holds. Let $a \in L \cap G \cap R$, where $L$ is a left ideal, $G$ is a generalized bi-ideal and $R$ is a right ideal of $S$, this implies that there exist elements $x, y \in S$ such that $a \leq\left(x a^{2}\right) y$. Now

$$
\begin{aligned}
a & \leq\left(x a^{2}\right) y=(x(a a)) y=(a(x a)) y=(y(x a)) a . \\
y(x a) & \leq y\left(x\left(\left(x a^{2}\right) y\right)\right)=y\left(\left(x a^{2}\right)(x y)\right)=\left(x a^{2}\right)(y(x y)) \\
& =\left(x a^{2}\right)\left(x y^{2}\right)=(x(a a)) m, \text { say } x y^{2}=m \\
& =(a(x a)) m=(m(x a)) a .
\end{aligned}
$$

Thus $a \leq\left(x a^{2}\right) y=(y(x a)) a=((m(x a)) a) a \in(L G) R$, i.e., $a \in((L G) R]$. Hence $L \cap G \cap R \subseteq$ $\subseteq((L G) R]$, i.e., $(1) \Rightarrow(4)$. (4) $\Rightarrow(3)$, every bi-ideal of $S$ is a generalized bi-ideal of $S$. (3) $\Rightarrow(2)$, every quasi-ideal of $S$ is a bi-ideal of $S$. Assume that (2) is true. Now

$$
\begin{aligned}
L \cap S \cap R & \subseteq((L S) R]=(((e L) S) R]=(((S L) e) R]=(((S L)(e e)) R] \\
& =(((S e)(L e)) R] \subseteq((S(L e)) R] \subseteq((S L) R] \subseteq(L R] . \\
& \Rightarrow L \cap R \subseteq(L R] .
\end{aligned}
$$

Hence $S$ is an intra-regular by the Theorem 5, i.e., $(2) \Rightarrow(1)$.

\section{Regular and Intra-regular Ordered AG-groupoids}

In this section, we characterize both regular and intra-regular ordered AG-groupoids by the properties of (left, right, quasi-, bi-, generalized bi-) ideals.

Theorem 9. Let $S$ be an ordered AG-groupoid with left identity $e$ such that $(x e) S=x S$ for all $x \in S$. Then the following conditions are equivalent.

(1) $S$ is a regular and an intra-regular.

(2) $\left(B^{2}\right]=B$ for every bi-ideal $B$ of $S$.

(3) $B_{1} \cap B_{2}=\left(B_{1} B_{2}\right] \cap\left(B_{2} B_{1}\right]$ for all bi-ideals $B_{1}, B_{2}$ of $S$.

Proof: Suppose that (1) holds and $B$ be a bi-ideal of $S$. Since $\left(B^{2}\right]=(B B] \subseteq(B]=B$. Let $a \in B$, this implies that there exists an element $x \in S$ such that $a \leq(a x) a$, also there exist elements $y, z \in S$ such that $a \leq\left(y a^{2}\right) z$. Now

$$
\begin{aligned}
a & \leq(a x) a \leq(a x)\left(\left(y a^{2}\right) z\right)=\left(\left(\left(y a^{2}\right) z\right) x\right) a . \\
\left(\left(y a^{2}\right) z\right) x & =(x z)\left(y a^{2}\right)=m\left(y a^{2}\right), \text { say } m=x z \\
& =m(y(a a))=m(a(y a))=a(m(y a)) \\
& \leq((a x) a)(m(y a))=((a x) m)(a(y a)) \\
& =((m x) a)(a(y a))=(\text { na })(a(y a)), \text { say } n=m x \\
& =((e n) a)(a(y a))=((a n) e)(a(y a)) \\
& =((a n) a)(e(y a))=((a n) a)(y a)=(s a)(y a), \text { say } s=a n \\
& =(a a)(y s)=(a a) t, \text { say } t=y s \\
& \leq(((a x) a) a) t=((a a)(a x)) t=(t(a x))(a a) \\
& =(a(t x))(a a)=(a w)(a a), \text { say } w=t x .
\end{aligned}
$$


Thus $a \leq\left(\left(\left(y a^{2}\right) z\right) x\right) a \leq((a w)(a a)) a \in((B S) B) B \subseteq B^{2}$, i.e., $a \in\left(B^{2}\right]$. So $B \subseteq\left(B^{2}\right]$, i.e., $\left(B^{2}\right]=B$. Hence $(1) \Rightarrow(3)$. Assume that $(2)$ is true. Let $B_{1}, B_{2}$ be bi-ideals of $S$, then $B_{1} \cap B_{2}$ be also a bi-ideal of $S$. Now $B_{1} \cap B_{2}=\left(\left(B_{1} \cap B_{2}\right)\left(B_{1} \cap B_{2}\right)\right] \subseteq\left(B_{1} B_{2}\right]$ and $B_{1} \cap B_{2}=\left(\left(B_{1} \cap B_{2}\right)\left(B_{1} \cap B_{2}\right)\right] \subseteq$ $\subseteq\left(B_{2} B_{1}\right]$, thus $B_{1} \cap B_{2} \subseteq\left(B_{1} B_{2}\right] \cap\left(B_{2} B_{1}\right]$. First of all we have to show that $\left(B_{1} B_{2}\right]$ is a bi-ideal of $S$. It is enough to show that $\left(\left(B_{1} B_{2}\right] S\right)\left(B_{1} B_{2}\right] \subseteq\left(B_{1} B_{2}\right]$. Now

$$
\begin{aligned}
\left(\left(B_{1} B_{2}\right] S\right)\left(B_{1} B_{2}\right] & =\left(\left(B_{1} B_{2}\right](S]\right)\left(B_{1} B_{2}\right] \\
& \subseteq\left(\left(B_{1} B_{2}\right) S\right]\left(B_{1} B_{2}\right] \\
& \subseteq\left(\left(\left(B_{1} B_{2}\right) S\right)\left(B_{1} B_{2}\right)\right] \\
& =\left(\left(\left(B_{1} B_{2}\right)(S S)\right)\left(B_{1} B_{2}\right)\right] \\
& =\left(\left(\left(B_{1} S\right)\left(B_{2} S\right)\right)\left(B_{1} B_{2}\right)\right] \\
& =\left(\left(\left(B_{1} S\right) B_{1}\right)\left(\left(B_{2} S\right) B_{2}\right)\right] \subseteq\left(B_{1} B_{2}\right] \\
& \left.\Rightarrow\left(\left(\left(B_{1} B_{2}\right) S\right)\left(B_{1} B_{2}\right)\right)\right] \subseteq\left(B_{1} B_{2}\right] .
\end{aligned}
$$

Thus $\left(B_{1} B_{2}\right]$ is a bi-ideal of $S$, similarly $\left(B_{2} B_{1}\right]$ is also a bi-ideal of $S$. Then $\left(B_{1} B_{2}\right] \cap\left(B_{2} B_{1}\right]$ is also a bi-ideal of $S$. Now

$$
\begin{aligned}
\left(B_{1} B_{2}\right] \cap\left(B_{2} B_{1}\right] & =\left(\left(\left(B_{1} B_{2}\right] \cap\left(B_{2} B_{1}\right]\right)\left(\left(B_{1} B_{2}\right] \cap\left(B_{2} B_{1}\right]\right)\right] \\
& \subseteq\left(\left(B_{1} B_{2}\right]\left(B_{2} B_{1}\right]\right] \subseteq\left(\left(\left(B_{1} B_{2}\right)\left(B_{2} B_{1}\right)\right]\right] \\
& \left.\left.=\left(\left(B_{1} B_{2}\right)\right) B_{2} B_{1}\right)\right] \subseteq\left(\left(B_{1} S\right)\left(S B_{1}\right)\right] \\
& =\left(\left(\left(S B_{1}\right) S\right) B_{1}\right]=\left(\left(\left((S e) B_{1}\right) S\right) B_{1}\right] \\
& =\left(\left(\left(\left(B_{1} e\right) S\right) S\right) B_{1}\right]=\left(\left(\left(B_{1} S\right) S\right) B_{1}\right] \\
& =\left(\left((S S) B_{1}\right) B_{1}\right]=\left(\left(S B_{1}\right) B_{1}\right]=\left(\left((S e) B_{1}\right) B_{1}\right] \\
& =\left(\left(\left(B_{1} e\right) S\right) B_{1}\right]=\left(\left(B_{1} S\right) B_{1}\right] \subseteq\left(B_{1}\right] \\
& \Rightarrow\left(B_{1} B_{2}\right] \cap\left(B_{2} B_{1}\right] \subseteq\left(B_{1}\right]=B_{1} .
\end{aligned}
$$

Similarly, we have $\left(B_{1} B_{2}\right] \cap\left(B_{2} B_{1}\right] \subseteq\left(B_{2}\right]=B_{2}$, thus $\left(B_{1} B_{2}\right] \cap\left(B_{2} B_{1}\right] \subseteq B_{1} \cap B_{2}$. Therefore $B_{1} \cap B_{2}=\left(B_{1} B_{2}\right] \cap\left(B_{2} B_{1}\right]$, i.e., $(2) \Rightarrow(3)$. Suppose that (3) holds, let $R$ be right ideal of $S$ and $I$ be an ideal of $S$. Then $R$ and $I$ be bi-ideals of $S$, because every right ideal and two sided ideal of $S$ is bi-ideal of $S$ by the Lemma 9 . Now $R \cap I=(R I] \cap(I R]$, this implies that $R \cap I \subseteq(R I] \cap(I R]$. Thus $R \cap I \subseteq(R I]$ and $R \cap I \subseteq(I R]$, where $I$ is also a left ideal of $S$. Since $(R I] \subseteq R \cap I$, i.e., $(R I]=R \cap I$, thus $S$ is a regular by the Theorem 1 . Also, $R \cap I \subseteq(I R]$, thus $S$ is an intra-regular by the Theorem 5. Hence $(3) \Rightarrow(1)$.

Theorem 10. Let $S$ be an ordered AG-groupoid with left identity $e$ such that $(x e) S=x S$ for all $x \in S$. Then the following conditions are equivalent.

(1) $S$ is regular and intra-regular.

(2) Every quasi-ideal of $S$ is an idempotent.

Proof: Suppose that (1) holds. Let $Q$ be a quasi-ideal of $S$ and $\left(Q^{2}\right]=(Q Q] \subseteq(Q]=Q$, i.e., $\left(Q^{2}\right] \subseteq Q$. Let $a \in Q$, this implies that there exists an element $x \in S$ such that $a \leq(a x) a$, also there exist elements $y, z \in S$ such that $a \leq\left(y a^{2}\right) z$. Now 


$$
\begin{aligned}
a & \leq(a x) a \leq(a x)\left(\left(y a^{2}\right) z\right)=\left(\left(\left(y a^{2}\right) z\right) x\right) a . \\
\left(\left(y a^{2}\right) z\right) x & =(x z)\left(y a^{2}\right)=m\left(y a^{2}\right), \text { say } m=x z \\
& =m(y(a a))=m(a(y a))=a(m(y a)) \\
& \leq((a x) a)(m(y a))=((a x) m)(a(y a)) \\
& =((m x) a)(a(y a))=(q a)(a(y a)), \text { say } q=m x \\
& =((e q) a)(a(y a))=((a q) e)(a(y a)) \\
& =((a q) a)(e(y a))=((a q) a)(y a)=(s a)(y a), \text { say } s=a q \\
& =(a a)(y s)=(a a) t, \text { say } t=y s \\
& \leq(((a x) a) a) t=((a a)(a x)) t=(t(a x))(a a) \\
& =(a(t x))(a a)=(a w)(a a), \text { say } w=t x
\end{aligned}
$$

Thus $a \leq\left(\left(\left(y a^{2}\right) z\right) x\right) a \leq((a w)(a a)) a \in((Q S) Q) Q \subseteq Q Q \subseteq Q^{2}$, i.e., $a \in\left(Q^{2}\right]$, because every quasi-ideal of $S$ is a bi-ideal of $S$ by the Lemma 12. Thus $Q \subseteq\left(Q^{2}\right]$, i.e., $\left(Q^{2}\right]=Q$. Hence $(1) \Rightarrow(2)$. Assume that (2) is true. Let $a \in S$, then $S a$ is a left ideal of $S$ containing $a$, so $S a$ is a quasi-ideal of $S$, because every left ideal of $S$ is a quasi-ideal of $S$. Now $S a=\left((S a)^{2}\right]=((S a)(S a)]$, i.e., $a \in((S a)(S a)]$. Thus $S$ is an intra-regular by the Theorem 5. Now $S a=((S a)(S a)]=(((S e) a)(S a)]=(((a e) S)(S a)]=$ $=((a S)(S a)]$, i.e., $a \in((a S)(S a)]$. Thus $S$ is a regular by the Theorem 1 . Therefore $(2) \Rightarrow(1)$.

Theorem 11. Let $S$ be an ordered AG-groupoid with left identity $e$ such that $(x e) S=x S$ for all $x \in S$. Then the following conditions are equivalent.

(1) $S$ is regular and intra-regular.

(2) Every quasi-ideal of $S$ is an idempotent.

(3) Every bi-ideal of $S$ is an idempotent.

Proof: $(1) \Rightarrow(3)$, by the Theorem 9 . (3) $\Rightarrow(2)$, every quasi-ideal of $S$ is a bi-ideal of $S$, by the Lemma 12. $(2) \Rightarrow(1)$, by the Theorem 10 .

Theorem 12. Let $S$ be an ordered AG-groupoid with left identity $e$ such that $(x e) S=x S$ for all $x \in S$. Then the following conditions are equivalent.

(1) $S$ is regular and intra-regular.

(2) $Q_{1} \cap Q_{2} \subseteq\left(Q_{1} Q_{2}\right]$ for all quasi-ideals $Q_{1}, Q_{2}$ of $S$.

(3) $Q \cap B \subseteq(Q B]$ for every quasi-ideal $Q$ and every bi-ideal $B$ of $S$.

(4) $B \cap Q \subseteq(B Q]$ for every bi-ideal $B$ and every quasi-ideal $Q$ of $S$.

(5) $B_{1} \cap B_{2} \subseteq\left(B_{1} B_{2}\right]$ for all bi-ideals $B_{1}, B_{2}$ of $S$.

Proof: Suppose that (1) holds. Let $B_{1}, B_{2}$ be bi-ideals of $S$, then $B_{1} \cap B_{2}$ be also a bi-ideal of $S$. Since every bi-ideal of $S$ is an idempotent by the Theorem 9 , then $B_{1} \cap B_{2}=\left(\left(B_{1} \cap B_{2}\right)^{2}\right]=$ $=\left(\left(B_{1} \cap B_{2}\right)\left(B_{1} \cap B_{2}\right)\right] \subseteq\left(B_{1} B_{2}\right]$. Hence $(1) \Rightarrow(5)$. Since $(5) \Rightarrow(4) \Rightarrow(2)$ and $(5) \Rightarrow(3) \Rightarrow(2)$, because every quasi-ideal of $S$ is a bi-ideal of $S$ by the Lemma 12. Assume that (2) is true. Now $R \cap L \subseteq(R L]$, where $R$ is a right ideal and $L$ is a left ideal of $S$. Since $(R L] \subseteq R \cap L$, i.e., $R \cap L=(R L]$, thus $S$ is regular. Again by (2) $L \cap R \subseteq(L R]$, thus $S$ is an intra-regular. Therefore $(2) \Rightarrow(1)$.

\section{Conclusion}

In this article, we have characterized the non-associative ordered semigroups in terms of their onesided ideals, ideals, interior ideals, bi-ideals and quais ideals. We have also characterized the intraregular and regular orderded AG-groupoids through the properties of their ideals. 


\title{
References
}

1 Kazim, M. A. \& Naseerudin, M. (1972). On almost semigroups. Alig. Bull. Math., 2, 1-7.

2 PV, P. (1995). AG-test and some general properties of Abel-Grassmann's groupoids. Pure Mathematics and Applications, 6, 4, 371-383.

3 Mushtaq, Q. \& Yusuf, S. M., (1978). On LA-semigroups. Alig. Bull. Math., 8, 65-70.

4 Jezek, J. \& Kepka, T. (1983). Medial groupoids. Rozpravy CSAV Rada Mat. a Prir. Ved, 93, 2, 93.

5 Cho, J. R., Jezek, J. \& Kepka, T. (1999). Paramedial groupoids. Czechoslovak Mathematical Journal, 49, 2, 277-290.

6 Kehayopulu, N. (1990). Remark on ordered semigroups. Math. Japon., 35, 6, 1061-1063.

7 Kehayopulu, N. (1990). On left regular ordered semigroups. Math. Japon., 35, 6, 1051-1060.

8 Kehayopulu, N. (1993, December). On intra-regular ordered semigroups. In Semigroup Forum, Springer-Verlag, 46, 1, 271-278.

9 Kehayopulu, N. (1997). On regular ordered semigroups. Mathematica japonicae, 45, 3, 549-553.

10 Kehayopulu, N. (1998). On completely regular ordered semigroups. Sci. Math, 1, 1, 27-32.

Н. Kaусар, М. Мунир, М. Гульзар, Г. М. Аддис

\section{Реттелген AG-группоидтардың әртүрлі идеалды кластарының қасиеттері бойынша сипаттамасы}

\begin{abstract}
Мақалада ассоциативті емес жартылай группалардың идеалдарына қатысты кейбір маңызды сипаттамалар ұсынылған. Біріншіден, біз реттелген AG-группоидты оның идеалының қасиеттері тұрғысынан сипаттадық, содан кейін осы AG-группоидтардың екі маңызды класына, яғни регулярлық және ішкі регулярлық емес ассоциативті емес AG-группоидтарға сипаттама бердік. Біздің мақсатымыз реттелген AG-группоид деп аталатын ассоциативті емес және коммутативті емес алгебралық құрылымдар класын зерттеу арқылы ассоциативті алгебралық құрылымдарды зерттеу мен дамытуды ынталандыру.
\end{abstract}

Kiлm сөздер: реттелген AG-группоидтар, солға (оң, ішкі, квази-, би-, жалпыланған би-) идеалдар, регулярлық (ішкі регулярлық) реттелген AG-группоидтар.

Н. Каусар, М. Мунир, М. Гульзар, Г. М. Аддис

\section{Характеризация упорядоченных AG-группоидов через свойства их различных классов идеалов}

\footnotetext{
В статье представлены некоторые важные характеристики упорядоченных неассоциативных полугрупп относительно их идеалов. Сначала были охарактеризован упорядоченный AG-группоид через свойства его идеалов, затем два важных класса этих AG-группоидов, а именно, регулярные и внутрирегулярные неассоциативные AG-группоиды. Цель настоящей работы - стимулирование исследования и развитие ассоциативных алгебраических структур путем изучения класса неассоциативных и некоммутативных алгебраических структур, называемых упорядоченным AG-группоидом.

Ключевые слова: упорядоченные AG-группоиды, левые (правые, внутренние, квази-, би-, обобщенные би-) идеалы, регулярные (внутрирегулярные) упорядоченные AG-группоиды.
} 